\title{
Detection of Novel Neutralizing Antibody Reactivities Against The Membrane Proximal External Region (MPER) of gp4 I in HIV-I Infected Humans
}

\section{George M Shaw*}

Address: Univ. of Alabama, B'ham, AL

Email: George M Shaw* - gshaw@uab.edu

* Corresponding author $¥$ Presenting author

\author{
from 2005 International Meeting of The Institute of Human Virology \\ Baltimore, USA, 29 August - 2 September 2005 \\ Published: 8 December 2005 \\ Retrovirology 2005, 2(Suppl I):SI02 doi:I0.1 I86/I742-4690-2-SI-SI02
}

\section{Background}

Previously, we employed site-directed mutagenesis to introduce HIV-1 4E10 and 2F5 epitopes into the corresponding region of a functional HIV-2 envelope glycoproteins (AIDS Vaccine 2005, abstract \#A210). The resulting "chimeric" viruses were used to screen HIV-1 infected human plasma for $4 \mathrm{E} 10$ or 2F5-like neutralizing antibodies (Nabs). Among 177 subjects infected with HIV-1 representing ten different subtypes or circulating recombinant forms, none had significant Nab titers directed toward these epitopes.

\section{Materials and methods}

Here, we tested the same HIV-1 positive plasma specimens for neutralizing activity against chimeric HIV-2 viruses in which we substituted the complete 25 amino acid HIV-1 MPER (designated clone C1) or non-overlapping amino-terminal or carboxy-terminal portions of it (designated $\mathrm{C} 3$ and $\mathrm{C} 4$, respectively) using a single-cycle infectivity assay (Nature 422:307, 2003).

\section{Results}

HIV-2 viruses containing the $\mathrm{C} 1, \mathrm{C} 3$ or $\mathrm{C} 4 \mathrm{MPER}$, and the parental virus HIV-2/7312A, were infectious and equally susceptible to neutralization by $\mathrm{T} 1249, \mathrm{sCD} 4$, the antiHIV-2 Env mAb 1.7A, and polyclonal anti-HIV-2 antibodies. This result demonstrates that none of the chimeric HIV-2 viruses was "globally sensitive" to neutralization. Surprisingly, 60 out of 165 (36\%) of HIV-1 plasmas tested contained MPER specific Nabs. IC50 titers ranged from 0.0005-0.02 (mean 0.006; median 0.004; standard deviation 0.004). Anti-MPER Nab reactivities were mapped to C3 (6 subjects) or C4 (14 subjects) regions of the HIV-1
MPER or to epitopes spanning them (13 subjects). None of the 60 subjects with Nabs to C1, C3, or C4 had antibodies that neutralized HIV-2 viruses containing $2 \mathrm{~F} 5$ or $4 \mathrm{E} 10$ epitopes only.

\section{Conclusion}

These results indicate that the MPER of HIV-1 elicits Nab responses in a substantial proportion of infected patients and that the epitopes recognized by these Nabs are distinct from those recognized by $4 \mathrm{E} 10$ or $2 \mathrm{~F} 5$. 